\title{
Evaluation of a Higher Education Institute's Quality Framework performance during COVID-19
}

\section{Paul O'Leary, Derek O'Byrne}

Office of the Registrar, Waterford Institute of Technology, Ireland.

\begin{abstract}
COVID-19 provided a challenge to the continuing high quality operation of higher education. Quality frameworks, which were created long before national lockdowns or social distancing, were tested in a manner that had not been foreseen on their creation.

This work examines the performance of the framework in our institute to see if it was sufficiently robust to offer our students a quality education experience, to propose some modifications and to reassure the public in terms of the standard of our graduates.

Engagement with the student body is described in detail from decision-making to evaluation at the end of the academic year of their experience on their programme of study.
\end{abstract}

Keywords: Quality framework; emergency teaching; student engagement. 


\section{Introduction}

On $12^{\text {th }}$ March 2020, the Irish government announced the closure of schools, pre-schools and further and higher education settings, initially until March $29^{\text {th }}$, to limit the spread of COVID19. To minimise the impact on teaching and learning, educators were asked to provide online resources for students or online lessons, while taking into account students that may not have access to online facilities. On $27^{\text {th }}$ March, the Irish government introduced a national lockdown, banning all non-essential travel and contact with people outside one's home (including family and partners).

This paper examines the impact in one Irish Higher Education institute of this dramatic change on the education offered and the manner in which the educational quality framework was tested and fared in completing Academic Year 2019-20 and then in delivering Academic Year 2020-21.

While the physical structures of the institute were closed, students, staff and the public had an expectation that the institute would continue to deliver education and to maintain the same high standards.

\section{Quality Framework}

The institute's quality framework was crucial to the successful negotiation of the national lockdown and the completion of the 2019-20 academic year. The quality framework preCOVID operated in an ever-increasing, complex environment that nonetheless provided for effective policies and procedures. Of course the framework was drawn up without ever anticipating the nature and extent of the complexity that subsequently ensued.

The institute's quality framework was tested in many areas, most notably:

- $\quad$ Academic and Student-centred Values

- Staff and Student Quality Culture

- Informed Practice and External Engagement

- Proportional Implementation of Quality Processes

- Comprehensive, Transparent and Publicly Accountable QA

- Consistency with Policy and International Effective Practice

- Measurement

\subsection{Academic and Student-centred Values}

One aspect of the first national lockdown is that the students were suddenly off-campus. The institute decided nonetheless to prioritise the students' perspective from the beginning, with the students union representing the student voice. The students union and institute codeveloped a COVID-19 Student Charter, which described the responsibilities and 
commitments of the student body, the institute and students union and explained what education would look like in the COVID-19 era. The charter particularly highlighted the need for strong communication links to all parties, which was tested two months later, in a survey of the entire student population. From the closure day on March $12^{\text {th }}$, the institute consistently engaged with the students union and listened to ideas, challenges and concerns. Adapting learning to a COVID-19 era was difficult, but it helped considerably to do so in a manner that was collaborative and engaging. However, survey results presented later in this research indicate that the communications to the wider student body need improvement.

The dynamic and complex nature of the COVID-19 challenge meant that some early decisions were later modified as more information came to light. For example, the Academic Council decided in May 2020 to hold semester 1 repeat examinations on campus as usual in August, to mirror the format of the original exams pre-pandemic. However, as the exam dates in August approached, some students expressed misgivings about coming on campus or their inability to travel due to a regional lockdown. Academic Council decided to continue with the examinations, but also that no student would be disadvantaged, as a result of their concerns about or restrictions on attending on-campus exams. Academic Council therefore established a mitigation process, which included the possibility of September alternative assessments and modified progression rules.

Finally in terms of academic-centric values, the institute has a core objective of recruiting and developing high-quality academic staff and researchers. Therefore training and guidelines were prepared to address the pressing needs of academic staff who had a dramatic change in their academic delivery and assessment. The dedicated Technology-Enhanced Learning unit significantly increased the number and nature of training workshops to support the academic staff development and the effective utilization of the eLearning infrastructure.

\subsection{Staff and Student Quality Culture}

The institute's Quality Culture under COVID-19 was successful due to three interrelated activities engaging staff, students and external stakeholders:

1. the sustained operation of cycles of development, implementation and review of policies and procedures that inform activity and initiate new policy, procedures and practices;

2. a defined structure that clearly articulates responsibility of key institute community members with respect to the quality assurance activities; and

3. continued publishing policies and procedures across the range of institute activities that were open for change by all stakeholders and eventually approved by the appropriate instructional body. 
Initially the organisation and communication of these activities was a challenge, especially as the institute community dealt with the infrastructure and broadband requirements of moving online. This quality culture was examined in a scheduled institutional review by an external panel of experts, with all participants communicating virtually. The original review was of course intended to be with the panel physically present on-campus, so there were concerns in relation to the operation of and capacity of a virtual review. However, notwithstanding the challenges and delays posed by COVID, the review meetings and communications before and after the external panel's virtual visit were well managed. The external panel examined the pre-COVID operation, the performance during the pandemic and the plans for the future. The panel's findings were broadly positive, but with recommendations to sustain ongoing improvements.

The scale and nature of the academic delivery and assessment changes were at levels heretofore never experienced. However, an example of the flexibility and resilience of the quality framework can be seen in the response to the challenge posed by students across the entire programme portfolio struggling to achieve all credits required for stage advancement, sometimes for reasons related to the pandemic, remote learning or regional lockdowns. Academic Council decided to delegate this key decision to each programme board. Therefore programme boards made decisions with students, in relation to students progressing, while carrying a larger than usually permitted number of credits. Programme Boards were empowered to permit students to progress carrying more than the previous maximum of 10 credits, subject to the Board being confident in the wisdom of each decision and to the Board informing the student of the challenge posed by carrying those modules.

\subsection{Informed Practice and External Engagement}

Engagement with all internal and external stakeholders is core to informing academic practice in learning, teaching, assessment and research; in collaboration; in economic and regional development; in establishing and realising civic and community objectives and in introducing and sustaining internationalisation.

When the first national lockdown occurred, the institute was already on a merger path with another regional institute, with a plan to then apply for a technological university status. This initiative is driven in part by many stakeholders in the region and continued notwithstanding the difficulties posed by the pandemic. The submission has been made to the government and an external review panel will begin its evaluation in mid-June.

\subsection{Proportional Implementation of Quality Processes}

The quality framework also includes the academic activities of the institute, including course design and approval, the setting and monitoring of standards, the making of awards and the academic integrity of the learner and learning process. Increasingly, these regulations must 
also account for different types of learners, including new modes of delivery (such as technology enhanced learning, including on-line delivery), as well as the diversity of types and backgrounds of learners.

It was vital therefore that the quality framework be dynamic, flexible and responsive in the pandemic period. For example, the validation of new awards was carried out online in a manner that met the needs of quality assurance and the expectations of the award proposers.

\subsection{Comprehensive, Transparent and Publicly Accountable $Q A$}

The validation and awarding procedures were still consistently comprehensive, transparent and publicly accountable, which for example meant that procedures and evaluations of new award proposals continued to be published online. Indeed online publication was arguably more important in this period of limited campus access, so the institute also published its own self-reflective report for the institutional review, as well as the expert panel report from that review. Previously the self-evaluation report had not been published.

\subsection{Consistency with Policy and International Effective Practice}

The institute is a full and committed members of the European Higher Education Area and, as such, benchmark their approach to quality assurance against the ESG and other comparable systems of international effective practice. The Quality Framework guiding principles, which form the core of this paper also define the basis for an annual quality review to the national statutory quality body. The annual report for the 2019-20 year detailed the effective performance of the framework, especially in the latter part of the year when the COVID response took hold.

\subsection{Monitoring and Measurement}

Measurement, reporting and publication are key aspects of good academic governance. The institute continued throughout the pandemic to measure and monitor performance, for example in new student applications, exam attendance, monitoring the transition online through indicators such as virtual learning environment page-hits and internal reviews of new and modified programmes.

Accountability, control and scrutiny were ensured through reporting, student surveys, programme and School Board reports, new programme and programme change panel review reports, postgraduate vivas, meeting the national statutory quality body in a Dialogue Meeting, and maintaining transparency of all framework actions through publication on the institute website.

Emergency Remote Teaching required an immediate and sustained response in the institute's Virtual Learning Environment, Moodle. Since March, Moodle has been available throughout, with $100 \%$ Moodle Uptime. Unsurprisingly, Moodle Traffic increased, up to $157 \%$ in the 
March-May period compared with similar months in 2019. The daily average of just under 60,000 Moodle pageviews in the March-May period has since was immediately exceeded on return to education in September 2020, where the resumption of classes saw the number of pageviews soar to new records of over 200,000 .

Student feedback and participation is an important aspect of the capacity of the institute to assess its performance. The entire student body was surveyed in May 2020, at the end of the 2019-2020 Academic Year. They gave a broadly positive response given on their experience on their programme in the previous year, notwithstanding the sudden move to emergency remote teaching and the dramatic changes in their assessment modes. Approximately $20 \%$ of the full-time major award students (about 1,300 students) took the survey. Notwithstanding all of the challenges that they faced since March, Figure 1 shows that the students' impression of their programme was better than the previous year's survey scores across every single category (scoring is on a Likert scale 1-4, with 4 being the highest rating).

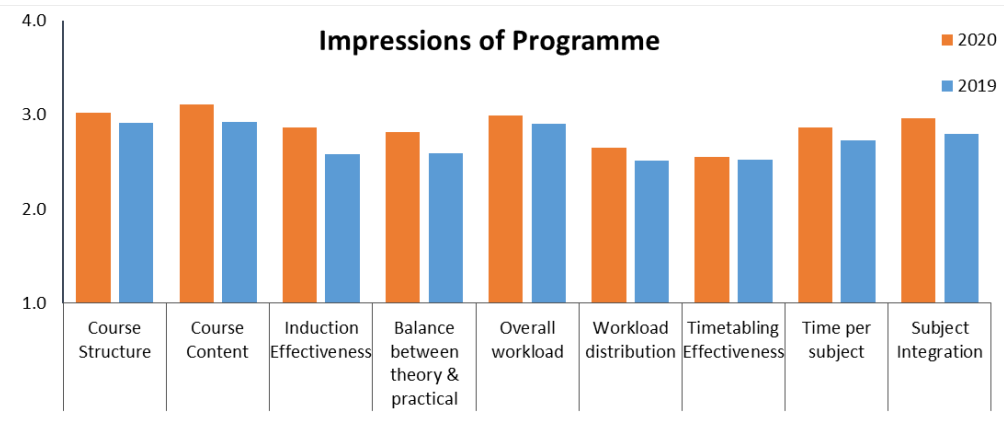

Figure 1. Students' impressions of their programme: 2020 versus 2019.

While this result is somewhat surprising a word analysis of the Course Strengths question in Figure 2 goes some way to explaining the students' rationale, where institute lecturers are clearly regarded highly by the students. 


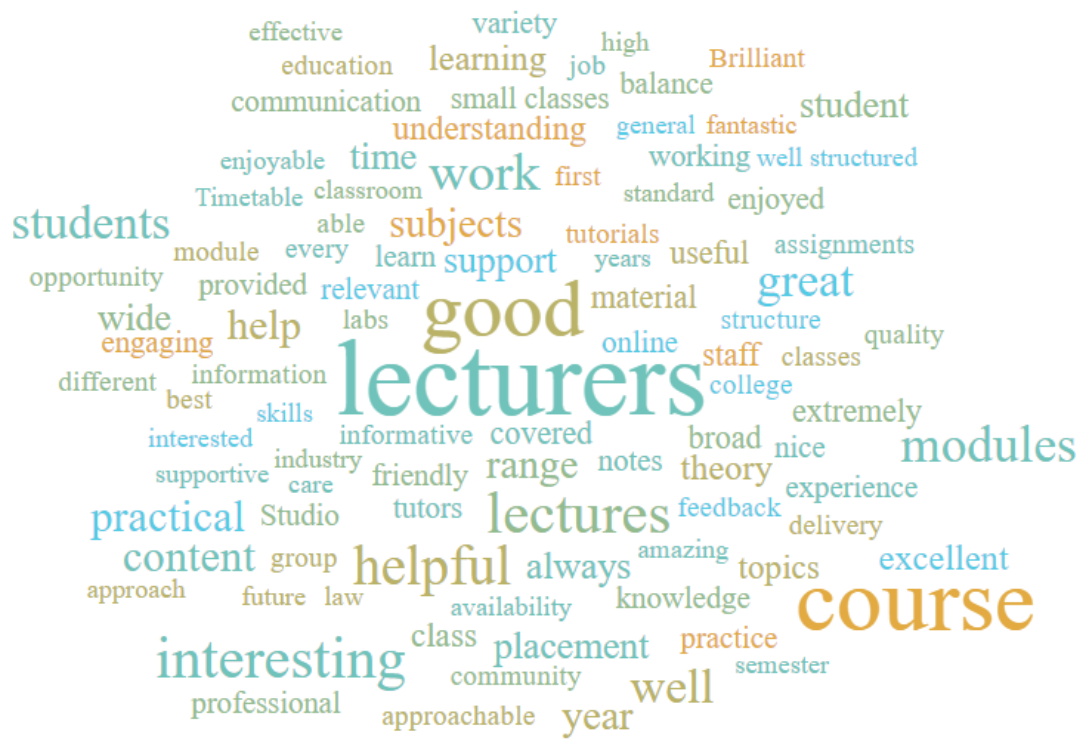

Figure 2. 2020 responses on course good features $(f=7)$.

However, in spite of improved efforts to communicate with students, the 2020 students ranked the effectiveness of communication as the lowest scoring theme in Figure 3 and, with the exception of Project Support and Continuous Assessment Feedback, ranked the questions in this category lower than the 2019 responding students. Furthermore, this section had been the strongest category of responses in the 2019 survey, so there is a clear message to the institute that communications must be improved. The institute has changed its communication approach for the academic year 2020-21.

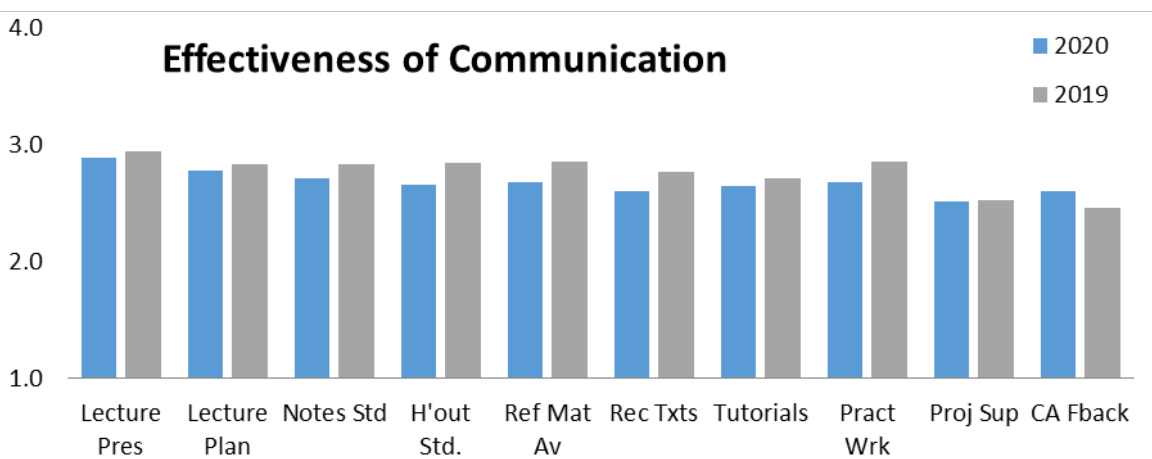

Figure 3. Effectiveness of communication: 2020 versus 2019. 
Many responding students have clear ideas on where their courses can be improved (setting aside the blank and no weaknesses responses), when responding to the Course Weaknesses question, and these are synthesised in Figure 4.

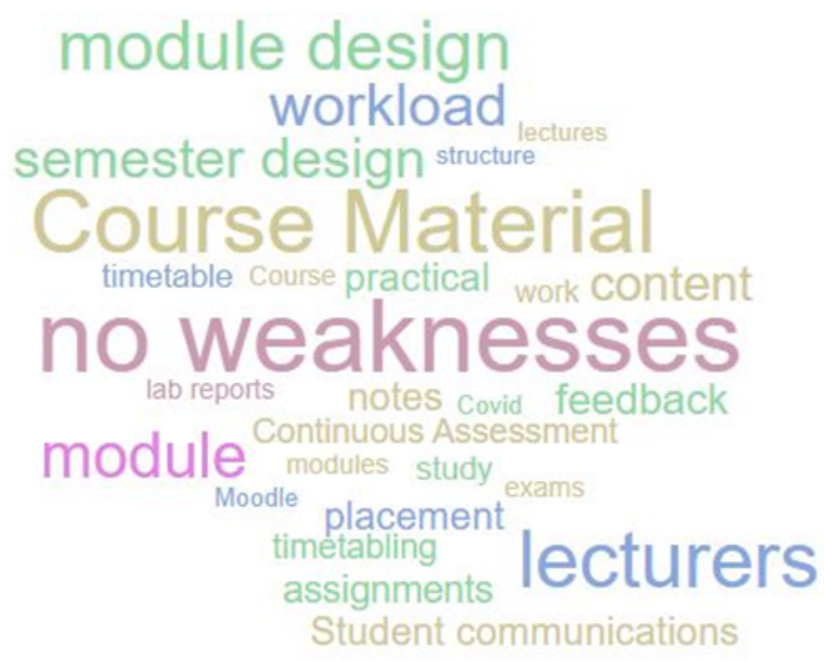

Figure 4. 2020 responses on course weaknesses $(f=6)$.

\section{Conclusion}

COVID-19 tested the quality framework of our higher education institute. However, the governance structure already in place was sufficiently robust to deal with all academic, regulatory and financial demands placed on it.

The institute student was involved in the solution from the outset, primarily through their representatives, in collaborative, engaged decision-making and student involvement in academic governance, but also through a heavily promoted survey for the entire student body to have their say. Overall, the students were happy with the institute's response, but expect communications to further improve.

The Quality Assurance Framework was not designed for a pandemic scenario but operated effectively during COVID-19. While the move to social distancing was initially a challenge, quite soon operations were continued as before, including new policy development; academic measurement, reporting and publication and oversight of emergency remote learning and teaching . 